\title{
Combined Analysis of Cosmic-Ray Anisotropy with IceCube and HAWC
}

\author{
The HAWC Collaboration ${ }^{1}$, The IceCube Collaboration ${ }^{2}$ \\ ${ }^{1}$ http://www.hawc-observatory.org/collaboration/icrc2017.php \\ ${ }^{2}$ http://icecube.wisc.edu/collaboration/authors/icrc17_icecube \\ E-mail: juan.diazvelez@alumnos.udg.mx
}

\begin{abstract}
During the past two decades, experiments in both the northern and southern hemispheres have observed a small but measurable energy-dependent sidereal anisotropy in the arrival direction distribution of Galactic cosmic rays with relative intensities at the level of one per mille. Individually, these measurements are restricted by limited sky coverage, and so the power spectrum of the anisotropy obtained from any one measurement displays a systematic correlation between different multipole modes $C_{\ell}$. We present the results of a joint analysis of the anisotropy on all angular scales using cosmic-ray data collected during 336 days of operation of the High-Altitude Water Cherenkov (HAWC) Observatory (located at $19^{\circ} \mathrm{N}$ ) and 5 years of data taking from the IceCube Neutrino Observatory (located at $90^{\circ} \mathrm{S}$ ) The results include a combined sky map and an all-sky power spectrum in the overlapping energy range of the two experiments at around 10 $\mathrm{TeV}$. We describe the methods used to combine the IceCube and HAWC data, address the individual detector systematics, and study the region of overlapping field of view between the two observatories.
\end{abstract}

Corresponding authors: J. C. Díaz-Vélez ${ }^{a, b *}$, M. Ahlers ${ }^{c}$, P. Desiati ${ }^{b}$, D. Fiorino ${ }^{d}$, ${ }^{a}$ Centro Universitario de los Valles, Universidad de Guadalajara, Guadalajara, Jalisco, México ${ }^{b}$ Wisconsin IceCube Particle Astrophysics Center (WIPAC) and Department of Physics, University of Wisconsin-Madison, Madison, WI 53706, USA

${ }^{c}$ Niels Bohr Institute, University of Copenhagen, Copenhagen, Denmark

${ }^{d}$ Department of Physics, University of Maryland, College Park, MD, USA

35th International Cosmic Ray Conference - ICRC2017

10-20 July, 2017

Bexco, Busan, Korea

* Speaker. 


\section{Introduction}

Over the last few decades, several studies have measured a small but significant variation in the intensity of cosmic rays of medium and high energies as a function of right ascension. This anisotropy was observed at energies of order $1 \mathrm{TeV}$ and higher by a number of experiments including Tibet AS $\gamma$ [1], Super-Kamiokande [2], Milagro [3], EAS-TOP [4], MINOS [5], ARGOYBJ [6], and HAWC [7] in the Northern Hemisphere and IceCube [8, 9, 10, 11] and its surface air shower array IceTop [12] in the Southern Hemisphere. In both hemispheres, the observed anisotropy has two main features: a large-scale structure with an amplitude of about $10^{-3}$, and a small-scale structure with an amplitude of $10^{-4}$ and angular size from $10^{\circ}$ to $30^{\circ}$.

A number of theories have proposed scenarios where the large-scale anisotropy results from the distribution of cosmic ray sources in the Galaxy and of their diffusive propagation $[2,3,7,10$, $11,13,14,15,16,17,18,19,20,21,22,23,24]$. However, the origin of the small-scale anisotropy is less well understood since it is expected that cosmic rays should lose any correlation with their original direction due to diffusion as they traverse through interstellar magnetic fields. There are several theories regarding the origin of this anisotropy, including structures in the heliomagnetic field, non-diffusive propagation, and turbulence in Galactic magnetic fields; see review in [25].

Analyses of data from Earth-based experiments with partial sky coverage suffer from systematic effects and statistical uncertainties of the calculated angular power spectrum. In this analysis we combine data from IceCube and HAWC at the same energy to study the full-sky anisotropy. Important information can be obtained from the power spectrum at low- $\ell$ (large scale), which is the region most affected by partial sky-coverage of one experiment only. However, it should be noted that neither observatory is sensitive to variations across declination bands. As a result, the dipole anisotropy can only be observed as a projection onto the equatorial plane.

\section{The Dataset}

Data selected for this analysis come from 5 years of IceCube data collected between May, 2011 and May, 2016, in its final configuration of 86 strings (IC86), as well as 1 year of HAWC data collected between April, 2015 and April, 2016, in its final configuration of 300 tanks (HAWC300). Table 1 shows the characteristics of both detectors next to each other. Only continuous days of data containing gaps of less than 20 min were chosen for these analyses in order to reduce the bias of uneven exposure along right ascension. There is also a difference in the median energy of the two experiments (Table 1). The median energy grows as a function of shower zenith angle and is largest in the narrow region of overlap between the two detectors at $\delta=-40^{\circ}$ to $-20^{\circ}$."

In order to select data that are consistent between the two detectors, we apply the following energy cuts: In the case of HAWC we use an energy reconstruction based on the likelihood method described in [26] to select events with energies at or above $10 \mathrm{TeV}$. We also apply a hadron/gamma separator described in [27] in order to exclude gamma-ray candidates. In the case of IceCube we apply a cut in the 2D plane of number of hit channels (which act as a proxy for muon energy) and the cosine of the zenith angle, as described in [11]. For a given number of hit channels, events at larger zenith angles are produced by cosmic-ray particles with higher energy. Figure 1a shows the distribution of data as a function of declination. The resulting energy distribution of the two 


\begin{tabular}{|c|c|c|c|c|}
\hline & \multicolumn{2}{|l|}{ IceCube } & \multicolumn{2}{|l|}{ HAWC } \\
\hline Hemisphere & \multicolumn{2}{|l|}{ Southern } & \multicolumn{2}{|l|}{ Northern } \\
\hline Latitude & \multicolumn{2}{|l|}{$-90^{\circ}$} & \multicolumn{2}{|l|}{$19^{\circ}$} \\
\hline Detection method & \multicolumn{2}{|c|}{ muons produced by $\mathrm{CR}$} & \multicolumn{2}{|c|}{ air showers produced by $\mathrm{CR}$ and $\gamma$} \\
\hline Field of view & \multicolumn{2}{|c|}{$-90^{\circ} /-20^{\circ}, \sim 4 \mathrm{sr}$ (same sky over $24 \mathrm{~h}$ ) } & \multicolumn{2}{|c|}{$-30^{\circ} / 64^{\circ}, \sim 2 \mathrm{sr}(8 \mathrm{sr}$ observed $) / 24 \mathrm{~h}$} \\
\hline Livetime & \multicolumn{2}{|l|}{5 years } & \multicolumn{2}{|c|}{269 days over a period of 336.36 days } \\
\hline \multirow[t]{2}{*}{ Detector trigger rate } & \multicolumn{2}{|l|}{$2.5 \mathrm{kHz}$} & \multicolumn{2}{|l|}{$25 \mathrm{kHz}$} \\
\hline & Quality cuts & Energy cuts & Quality cuts & Energy cuts \\
\hline Median primary energy & $20 \mathrm{TeV}$ & $10 \mathrm{TeV}$ & $2 \mathrm{TeV}$ & $10 \mathrm{TeV}$ \\
\hline Approx. angular resolution & $2^{\circ}-3^{\circ}$ & $2^{\circ}-6^{\circ}$ & $0.3^{\circ}-1.5^{\circ}$ & $0.3^{\circ}-1.5^{\circ}$ \\
\hline Events & $2.8 \times 10^{11}$ & $1.7 \times 10^{11}$ & $2.6 \times 10^{10}$ & $4.4 \times 10^{9}$ \\
\hline
\end{tabular}

Table 1: Comparison of the IceCube and HAWC datasets.

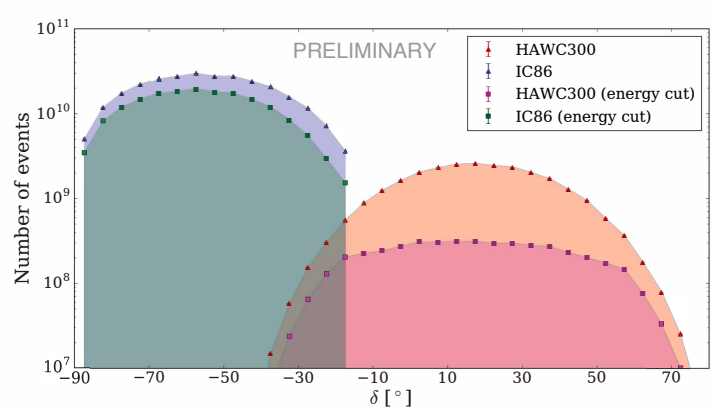

(a)

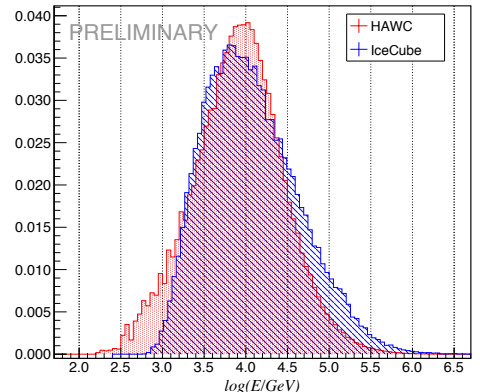

(b)

Figure 1: (a) Distribution of events as as a function of declination for IceCube and HAWC. Triangles correspond to the full energy spectrum and squares correspond to the same datasets after applying energy cuts. Restricting datasets to overlapping energy bins significantly reduces statistics for HAWC. The statistics in HAWC-300 before cuts are comparable to one year of IC86. (b) Energy distribution in each of the two datasets after cuts.

datasets is shown in Figure 1b. After cuts, both CR data sets have a median energy of approximately $10 \mathrm{TeV}$ with little dependence on zenith angle.

\section{Analysis}

We compute the relative intensity as a function of equatorial coordinates $(\alpha, \delta)$ by binning the sky into an equal-area grid with a bin size of $0.9^{\circ}$ using the HEALPix library [28]. The relative intensity gives the amplitude of deviations from the isotropic expectation in each angular bin $i$. In order to produce residual maps of the anisotropy of the arrival directions of the cosmic rays, we must have a description of the arrival direction distribution if the cosmic rays arrived isotropically at Earth, $\langle N\rangle(\alpha, \delta)_{i}$. Ground-based experiments observe CRs indirectly by detecting the secondary air shower particles produced by collisions of the cosmic-ray primary in the atmosphere. Simulations are not sufficiently accurate to describe the detector exposure at the level of $10^{-3}$. We therefore calculate this expected flux from the data themselves in order to account for rate variations in both time and viewing angle.

A common approach is to estimate the relative intensity and detector exposure simultaneously using time-integration methods such as time-scrambling [29] and direct-integration [30]. However, 
these methods can lead to an under- or overestimation of the isotropic reference level for detectors located at middle latitudes, because a fixed position on the celestial sphere is only observable over a relatively short period every day. The total number of cosmic ray events from this fixed position can only be compared against reference data observed during the same period. Therefore, time-integration methods can strongly attenuate large-scale structures exceeding the size of the instantaneous field of view [31]. In the next section we describe a likelihood method that can recover the full amplitude of the large-scale anisotropies projected on to the equatorial plane.

\subsection{Maximum likelihood method}

We apply the likelihood-based reconstruction developed by Ahlers et al. [31], generalized for combined anisotropy studies of data sets from multiple observatories that are exposed to overlapping regions of the sky. This likelihood method can disentangle the anisotropy from detector effects and gives a better estimate of the relative intensity of the sidereal cosmic-ray anisotropy for detectors in the middle latitudes (such as HAWC). The cosmic ray flux can then be expressed as $\phi(\alpha, \delta)=\phi^{\text {iso }} I(\alpha, \delta)$, where $I(\alpha, \delta)$ is the relative intensity as a function of position in the sky and $\phi^{\text {iso }}$ is the isotropic angular-averaged flux. Given that cosmic rays have been observed to be mainly isotropic, the flux is dominated by the isotropic term and therefore the anisotropy $\delta I=I-1$ is small.

For each observatory, the number of cosmic rays expected from an angular element of the local coordinate sphere $\Delta \Omega_{i}$ corresponding to coordinates $\left(\theta_{i}, \varphi_{i}\right)$ in a sidereal time interval $\Delta t_{\tau}$ is

$$
\mu_{\tau i} \simeq I_{\tau i} \mathscr{N}_{\tau} \mathscr{A}_{i}
$$

where $\mathscr{N}_{\tau}$ gives the expected number of isotropic events in sidereal time bin $\tau$ independent of pixel, $\mathscr{A}_{i}$ is the relative acceptance of the detector for pixel $i$, and $I_{\tau i}$ is the relative intensity observed in the local coordinates during time bin $\tau$. The likelihood of observing $n$ cosmic rays is then given by the product of Poisson probabilities

$$
\mathscr{L}(n \mid I, \mathscr{N}, \mathscr{A})=\prod_{\tau i} \frac{\left(\mu_{\tau i}\right)^{n_{\tau i}} e^{-\mu_{\tau i}}}{n_{\tau i} !},
$$

where $n_{\tau i}$ is the number of events observed in the local pixel $i$ during time bin $\tau$. We maximize the likelihood ratio of signal over null hypothesis in $\mathscr{N}, \mathscr{A}$, and $I$,

$$
\lambda=\frac{\mathscr{L}(n \mid I, \mathscr{N}, \mathscr{A})}{\mathscr{L}\left(n \mid I^{(0)}, \mathscr{N}^{(0)}, \mathscr{A}^{(0)}\right)} .
$$

The maximum $\left(I^{\star}, \mathscr{N}^{\star}, \mathscr{A}^{\star}\right)$ of the likelihood ratio (Eq. 3.3) results in a nonlinear set of equations that cannot be solved in an explicit form, but one can iteratively approach the best-fit solution.

In this combined analysis of HAWC and IceCube data, the likelihood (Eq. 3.2) is generalized to a product over data sets with individual detector exposures but the same relative intensity. This is a valid approach, as long as the rigidity distributions of the data sets are very similar. Systematic uncertainties caused by differences in energy and composition between the two data sets are currently being evaluated. Our reconstruction method is a simple generalization of the iterative method outlined in Ahlers et al. [31], where now the relative acceptances $\mathscr{A}$ and isotropic expectation $\mathscr{N}$ for each detector are evaluated as independent quantities. 


\subsection{Application of Method to Combined Dataset}

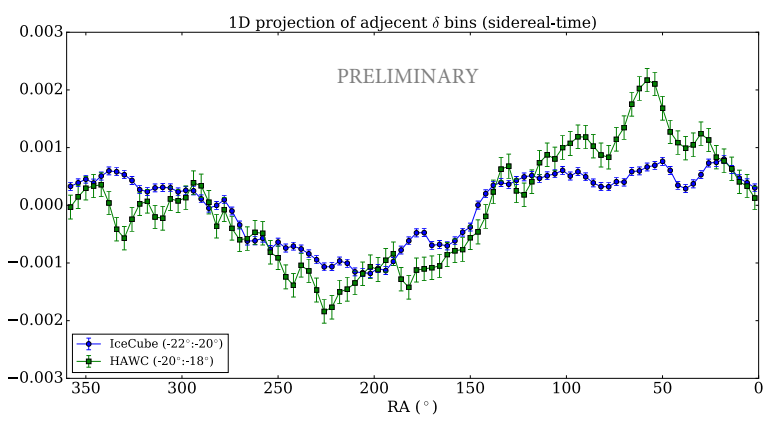

Figure 2: One-dimensional RA projection of the relative intensity of cosmic rays for adjacent $\delta$ bins at $-20^{\circ}$ for HAWC-300 and IC86 data. There is general agreement for large scale structures. The two curves correspond to different $\delta$ bands but some differences in the small scale structure might also be attributed to mis-reconstructed events that migrate from nearby $\delta$ bins with larger statistics. The errors are only statistical and don't account for systematic uncertainties.

Figure 2 shows the one-dimensional RA projection of the relative intensity of cosmic rays for adjacent $\delta$ bins at $-20^{\circ}$ for HAWC-300 and IC86 data. There is no overlapping region where the quality of the data is sufficient to allow a meaningful comparison so we have chosen two adjacent bins. The large structure between the two datasets is consistent though small structures differ. While HAWC data has a smaller point spread function and is sensitive to structures on smaller scales, IceCube has better statistics so the structures are more significant. One particular feature that stands out is the excess in HAWC around $\alpha=50^{\circ}$ that coincides with the so called region $A$. There appears to be a corresponding small excess in the IceCube data. It is worth noting that statistics in this region are quickly decreasing with increasing zenith angle, towards the horizon of each detector, as is the quality of angular reconstructions.

The relative intensity map obtained after 20 iterations of the maximum likelihood method is shown in Figure 3a. The corresponding statistical significance is obtained using a generalized form of the method of [32] and is shown in 3b. The significance of features in the northern sky is lower than previously published HAWC results due to decreased statistics given the energy cuts in this analysis. A smoothing procedure has been applied to all maps using a top-hat function in which a single pixel's value is the sum of all pixels within a $5^{\circ}$ radius. The significance of the IceCube region reflects the much larger number of statistics available in 5 years of IceCube as compared to 1 year of HAWC-300 at energies of $\sim 10 \mathrm{TeV}$. In order to eliminate larger structures, fitted multipoles can be subtracted to access lower angular scales while preserving the maximum angular scale throughout the map. Figure $3 \mathrm{c}$ shows the multipole fit with $\ell=\{1,2,3\}$ for the combined IC86 and HAWC-300 dataset. Figure $3 \mathrm{~d}$ is the residual relative intensity after subtracting the fitted multipole of $3 \mathrm{c}$ from the large-scale map in $3 \mathrm{a}$, and $3 \mathrm{e}$ is the corresponding statistical significance.

Figure $4 \mathrm{a}$ shows the angular power spectrum of the resulting relative intensity of cosmic rays for the combined IceCube and HAWC dataset calculated with the method described in [11] and [7]. The large scale structure corresponds to the peak in small $\ell$ while significant smaller structures up to $\ell \sim 10$ of angular scales between $10^{\circ}$ and $35^{\circ}$ can be seen above the gray band. The red dots correspond to the power spectrum resulting from subtracting the fitted multipoles $(\ell=1,2$, 


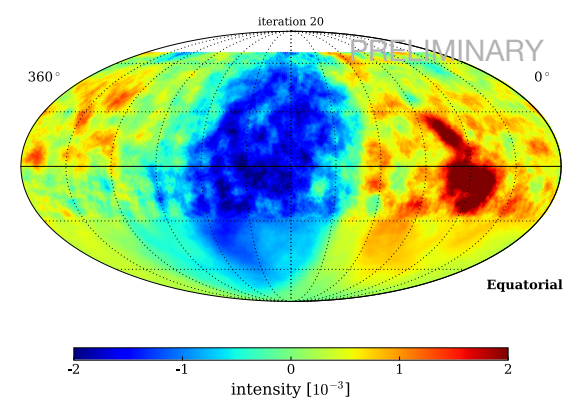

(a)

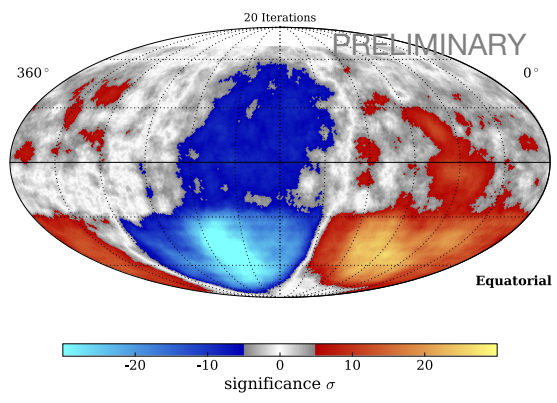

(b)

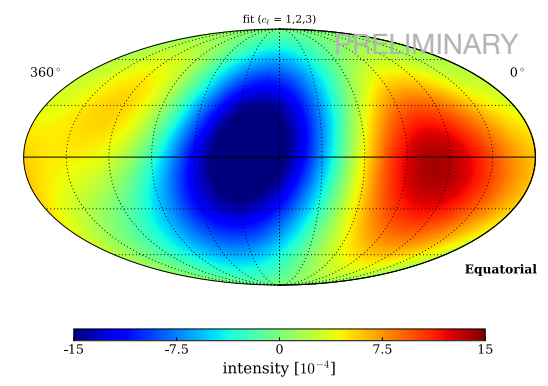

(c)

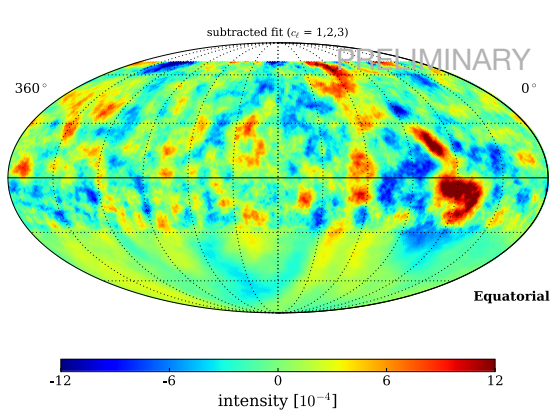

(d)

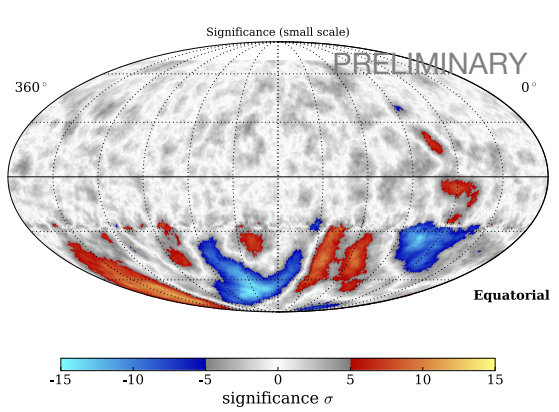

(e)

Figure 3: (a) Relative intensity from the maximum likelihood method after 20 iterations, (b) corresponding statistical significance, and (c) multipole fit with $\ell=\{1,2,3\}$ for the combined IC86 and HAWC-300 dataset. The significance of the IceCube region reflects the much larger statistics available in 5 years of IceCube compared to 1 year of HAWC-300 at energies of $\sim 10 \mathrm{TeV}$. (d) Relative intensity after subtracting the multipole fit from the large-scale map, and (e) the corresponding statistical significance.

3) and removing the large scale features. In Figure $4 \mathrm{~b}$ the likelihood method (stars) reconstructs a stronger dipole $(\ell=1)$ component compared to using direct integration over $24 \mathrm{~h}$ (squares). The full-sky coverage also provides better constraints for fitting the quadrupole $(\ell=2)$ and octupole $(\ell=3)$ components and reduces cross-talk between spherical harmonic expansion coefficients $a_{\ell m}$. Figure 5 shows the reconstructed dipole phase and amplitude from this analysis in the equatorial plane along with data from several other experiments (from [25]).

\section{Conclusions and Discussion}

The analysis of five years of data taken with the IceCube detector and 1 year of HAWC shows 


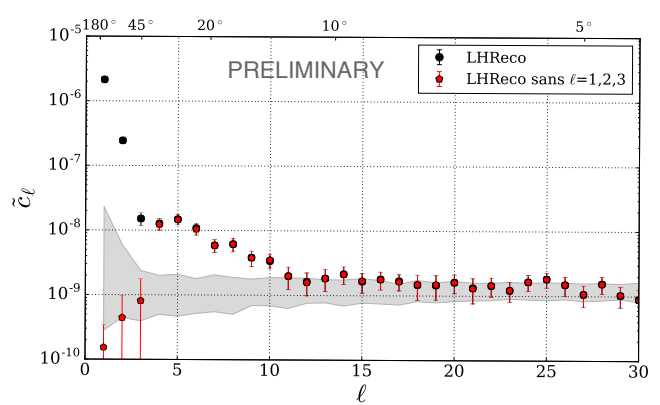

(a)

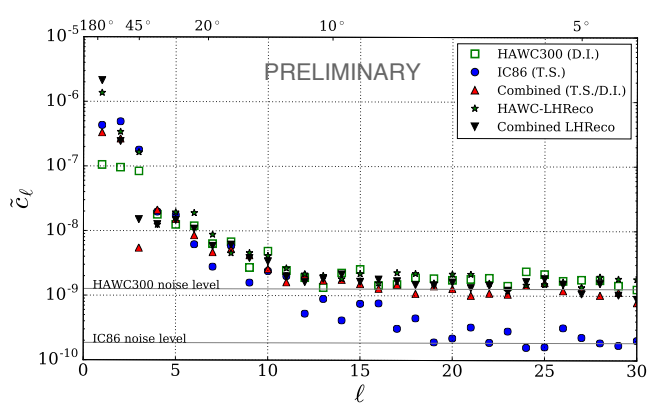

(b)

Figure 4: The angular power spectrum of the cosmic ray anisotropy (a) for the combined IceCube and HAWC dataset, and (b) comparison of power-spectra for IceCube and HAWC individually, and combined with time-integration and iterative methods. The red dots in (a) correspond to the power spectrum resulting from subtracting the fit to the large scale features $(\ell=1,2,3)$. The gray band represents the power spectra for isotropic sky maps at the $90 \%$ confidence level. The large scale structure corresponds to the peak in small $\ell$ on the left while significant smaller structures up to $\ell \sim 10$ of angular scales between $10^{\circ}$ and $35^{\circ}$ can be seen above the gray band. The noise level in the combined dataset is dominated by limited statistics for the portion of the sky observed by HAWC.

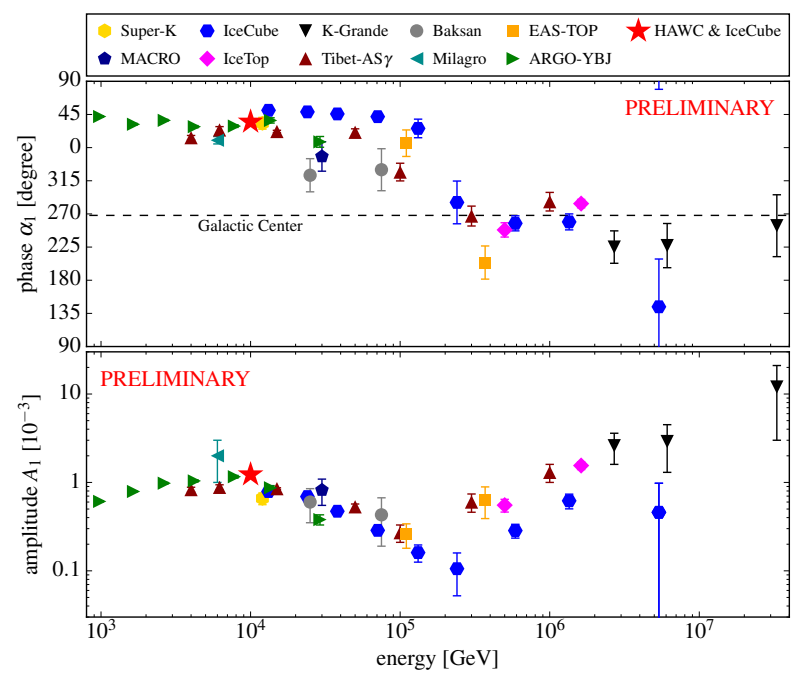

Figure 5: Summary plot (adopted from Ref. [25]) of the reconstructed TeV-PeV dipole amplitude and phase (see Ref. [25] for details and references).

an anisotropy in the arrival direction distribution of $10 \mathrm{TeV}$ cosmic rays that extends across both hemispheres. In this analysis we have used an iterative maximum-likelihood reconstruction method that simultaneously fits cosmic ray anisotropies and detector acceptance. The method does not rely on detector simulations and provides an optimal anisotropy reconstruction and the recovery of the dipole anisotropy for ground-based cosmic ray observatories located in the middle latitudes such as HAWC. Ground-based observatories are generally insensitive to cosmic-ray anisotropy variations that are symmetric in right ascension, i.e. only vary across declination bands. In particular, the dipole anisotropy can only be observed as a projection onto the celestial equator. The combined dataset provides almost full coverage of the sky and provides a better fit for the phase and 
amplitude of the horizontal component of the dipole anisotropy. In addition to a large-scale structure, we observe significant small-scale structure that is largely consistent with previous individual measurements.

\section{References}

[1] Tibet AS- $\gamma$ Collaboration, M. Amenomori et al., Astrophys. J. Lett. 626 (2005) L29-L32.

[2] Super-Kamiokande Collaboration, G. Guillian et al., Phys. Rev. D 75 (2007) 062003.

[3] Milagro Collaboration, A. A. Abdo et al., Phys. Rev. Lett. 101 (2008) 221101.

[4] M. Aglietta et al., Astrophys. J. Lett., 692 (2009) L130-L133.

[5] J. De Jong, ICRC2011 4 (2011) 46.

[6] ARGO-YBJ Collaboration, G. Di Sciascio, Euro. Phys. J. Web of Conf. 52 (2013) 4004.

[7] HAWC Collaboration, A. U. Abeysekara et al., Astrophys. J. 796 (2014) 108.

[8] IceCube Collaboration, M. G. Aarsen et al., JINST 12 (2017) P03012.

[9] IceCube Collaboration, R. Abbasi et al., Astrophys. J. Lett. 718 (2010) L194-L198.

[10] IceCube Collaboration, R. Abbasi et al., Astrophys. J. 740 (2011) 16.

[11] IceCube Collaboration, R. Abbasi et al., Astrophys. J. 746 (2012) 33.

[12] IceCube Collaboration, M. G. Aartsen et al., Astrophys. J. 765 (2013) 55.

[13] Tibet AS- $\gamma$ Collaboration, M. Amenomori et al., Science 314 (2006) 439-443.

[14] Milagro Collaboration, A. A. Abdo et al., Astrophys. J. 698 (2009) 2121-2130.

[15] Tibet AS- $\gamma$ Collaboration, M. Amenomori et al., Astropart. Phys. 36 (2012) 237-241.

[16] ARGO-YBJ Collaboration, B. Bartoli et al., Phys. Rev. 88 (2013) 082001.

[17] ARGO-YBJ Collaboration, B. Bartoli et al., Astrophys. J. 809 (2015) 90.

[18] A. D. Erlykin and A. W. Wolfendale, Astropart. Phys. 25 (2006) 183-194.

[19] P. Blasi and E. Amato, JCAP 1 (2012) 11.

[20] V. Ptuskin, Astropart. Phys. 39 (2012) 44-51.

[21] M. Pohl and D. Eichler, Astrophys. J. 766 (2013) 4.

[22] L. G. Sveshnikova, O. N. Strelnikova, and V. S. Ptuskin, Astropart. Phys. 50 (2013) 33-46.

[23] R. Kumar and D. Eichler, Astrophys. J. 785 (2014) 129.

[24] P. Mertsch and S. Funk, Phys. Rev. Lett. 114 (2015) 021101.

[25] M. Ahlers and P. Mertsch, Prog. Part. Nucl. Phys. 94 (2017) 184-216.

[26] HAWC Collaboration, A. U. Abeysekara et al, POS ( ICRC2017) 501 (these proceedings).

[27] HAWC Collaboration, A. U. Abeysekara et al., arXiv: 1701.01778.

[28] K. M. Górski et al., Astrophys. J. 622 (2005) 759-771.

[29] D. E. Alexandreas et al., Nucl. Instrum. Meth. A 328 (May, 1993) 570-577.

[30] Milagro Collaboration, R. W. Atkins et al., Astrophys. J. 595 (2003) 803-811.

[31] M. Ahlers et al., Astrophys. J. 823 (2016) 10.

[32] T.-P. Li and Y.-Q. Ma, Astrophys. J. 272 (1983) 317-324. 